\title{
Role of Boundaries in Control of Deformation Rate and Strength of Crystalline Materials
}

\author{
This paper is dedicated to Prof. Enrico Evangelista in the occasion of his $70^{\text {th }}$ birthday \\ W. Blum
}

University of Erlangen-Nürnberg, Dep. of Materials Science,

Martensstr. 5, 91058 Erlangen, Germany

wolfgang.blum@ww.uni-erlangen.de

\begin{abstract}
Keywords: Deformation Resistance, Flow Stress, Creep Rate, Steady State Deformation, Dislocation Structure, Generation of Dislocations, Annihilation of Dislocations, Low-angle Boundaries, High-angle Boundaries, Phase Boundaries, Nanostructured Materials
\end{abstract}

\begin{abstract}
Plastic deformation of crystalline materials is not controlled by interaction among free dislocations only, but the interaction of free dislocations with internal boundaries. i) Low-angle boundaries: Modeling of deformation of pure materials with conventional grain size on the basis of structure evolution indicates that low-angle boundaries act as obstacles of free dislocations. The migration of the low-angle boundaries constitutes an essential recovery process determining the deformation resistance in the steady state. ii) High-angle boundaries: Severe plastic deformation transforms low-angle boundaries into high-angle ones. They differ in obstacle and recovery characteristics from low-angle boundaries, which explains the special properties of ultrafine-grained and nanocrystalline materials with regard to strength, strain rate sensitivity and ductility. iii) Phase boundaries in Ni-base superalloys enhance the strengthening by hard phases with strengthening by dense dislocation networks serving to reduce coherency stresses. It is concluded that internal boundaries play a crucial role in controlling the evolution of structure and strength in crystalline materials.
\end{abstract}

\section{Introduction}

As a matter of fact, the plasticity of crystalline materials, i.e. their strength and deformability, depends on the defect structure. The most important defects are the dislocation lines, bounding the areas where crystallographic slip has occurred. Descriptions of plasticity are usually based on the average volumetric density $\rho$ of dislocation lines. However, considering individual dislocations is not enough. The long-range stresses carried by the dislocations lead to the emergence of a pattern of crystal boundaries made up by dislocations.

At the beginning of deformation, where glide is still relatively homogeneous on each slip system, the boundary dislocations are mainly in dipolar configuration. Both the formation of a dipole and its dissolution reduce the dislocation energy which is stored in the crystals. The process of dipole dissolution is tantamount to coalescence of neighboring slipped areas of the same type. Instead of distributing the dipoles evenly throughout the volume, it is energetically more favourable to deposit them in cell walls to clear the cell interiors from obstacles to glide. The dislocations gliding through the cell interior sweep the dipoles into the boundaries [1]. The spacing of the cell boundaries scales with the mean free path of the dislocations [2,3] (ratio of slipped area per stored dislocation length, see e.g. [4]).

Later in the deformation process the boundaries gradually take the character of low-angle boundaries. These consist of surplus dislocations of a given Burgers vectors which have like sign and therefore are unable to form dipoles. The surplus dislocations reduce their energies by forming low-angle boundaries. In a first step a group of surplus edge dislocations rearranges to form a finite wall. This saves energy as the compressive and tensile stresses below and above the edge dislocations partially compensate. However, the long-range stress fields are not affected by formation of a finite wall (which may be regarded as a dipole of two disclinations). They may, 
however, be relaxed by slip on other systems $[5,6]$. The surplus dislocations from these systems connect the free ends of the finite walls and thus lead to subgrains which are completely surrounded by boundaries and which have been able to rotate relative to their neighbors to relieve the longrange stresses. Another energetical advantage driving subgrain formation [7] is that the glide activities of the different slip systems are concentrated in different subgrains. This facilitates the glide inside a subgrain because the density of strong obstacles in the form of forest dislocations is reduced.

In addition to cell and low-angle subgrain boundaries, high-angle boundaries and phase boundaries need to be considered, as all types of boundaries constitute obstacles to the glide of individual dislocations. High-angle boundaries comprise the original grain boundaries, which extend in area as monotonic deformation goes on, and former low-angle boundaries, whose misorientation has crossed the value, where the boundary dislocations lose their identity because their cores overlap. The rate, at which the misorientation of low-angle boundaries increases with strain, depends on the behavior of the neighboring subgrains. When the sets of slip systems of the neighbors are different, the rate of increase of misorientation is particularly large [8]. New slip systems generate new types of boundaries. These facts play a role in generating ultrafine-grained materials by severe plastic deformation. Phase boundaries are existing in the vast majority of industrial materials which contain particles of foreign phases for reasons of strength or functional properties.

The present paper describes the influence of the boundaries on gliding dislocations. In principle, there are two opposing influences, as boundaries may act as dislocation obstacles as well as sinks for dislocations. It will be shown that the type of boundaries is of decisive influence on the resistance of crystalline materials to plastic deformation.

\section{Low-angle boundaries}

Under normal conditions of hot working and creep most of the subgrain boundaries are of lowangle type, with few exceptions of some boundaries of subgrains ending at a high-angle grain boundary of a polycrystal or at the surface. The low-angle character in part results from the fact that the strains involved are usually quite limited, in particular in creep, so that even boundaries separating regions of different slip-system choice do not have the chance to overcome the misorientation limit for low-angle boundaries lying in the region of $15^{\circ}$ (known from the calculation of dislocation spacings in low-angle boundaries and from observations of abrupt changes of boundary properties [9]).

In many models of deformation at high homologous temperature the low-angle boundaries are neglected. Deformation is often classified as climb-controlled or glide-controlled [10] with the idea in mind that edge dislocations need to climb in order to annihilate together with their dipole partner (recovery control) or that glide is the controlling process rather than climb as solutes attached to the edge dislocations limit their glide velocity to the extent that recovery is no longer controlled by the annihilation of dipoles, but by their formation. This classification is useful (see e.g. [2]), but not sufficient for detailed modeling. In the treatments of recovery by Kocks and Mecking [11] and Nes $[3,12]$ all the dislocations are assumed to take part in the recovery process. For instance, Nes and coworkers formulate the rate of dislocation annihilation as $\dot{\rho}^{-}=\rho v$ where $\rho$ is the total dislocation density and $v$ is the frequency at which it recovers by annihilation. However, only those dislocations can annihilate (and cause local coalescence of slipped areas) which are in a stable dipolar configuration. Therefore the density $\rho_{\text {dip }}$ of dislocation dipoles, i.e. the length of dislocations which is stored in dipolar configuration, needs to be considered as a structure parameter entering the expression for dislocation recovery by annihilation: $\dot{\rho}^{-}=\rho_{\text {dip }} v_{\text {dip }}[4,13]$.

Treating recovery of dipoles under the assumption of spatially homogeneous glide on the active slip systems $[4,13,14]$ leads to a rate $\dot{\varepsilon}_{\text {s }}$ of steady-state deformation as function of stress $\sigma$ and temperature $T$ which reproduces some of the experimentally observed features. This is 
demonstrated in the normalized plot of Fig. 1; normalization was achieved using the shear modulus $G$ [15], the coefficient of lattice self diffusion $D_{\mathrm{SD}}$ [15], and the length $b$ of the Burgers vector; $k_{\mathrm{B}}$ is the Boltzmann constant. The steady-state deformation rate is overestimated in the limiting case of low stresses $\sigma \approx 10^{-5} G$ by a factor of about 50 . This is no surprize as low-angle boundaries have not been taken into account in the model [14]. In fact, the gap between model and experimental data shrinks significantly at low stresses, if one looks at the creep rate at the point where the density $\rho_{\mathrm{f}}$ of free dislocations is first established while the subgrains have not yet formed.

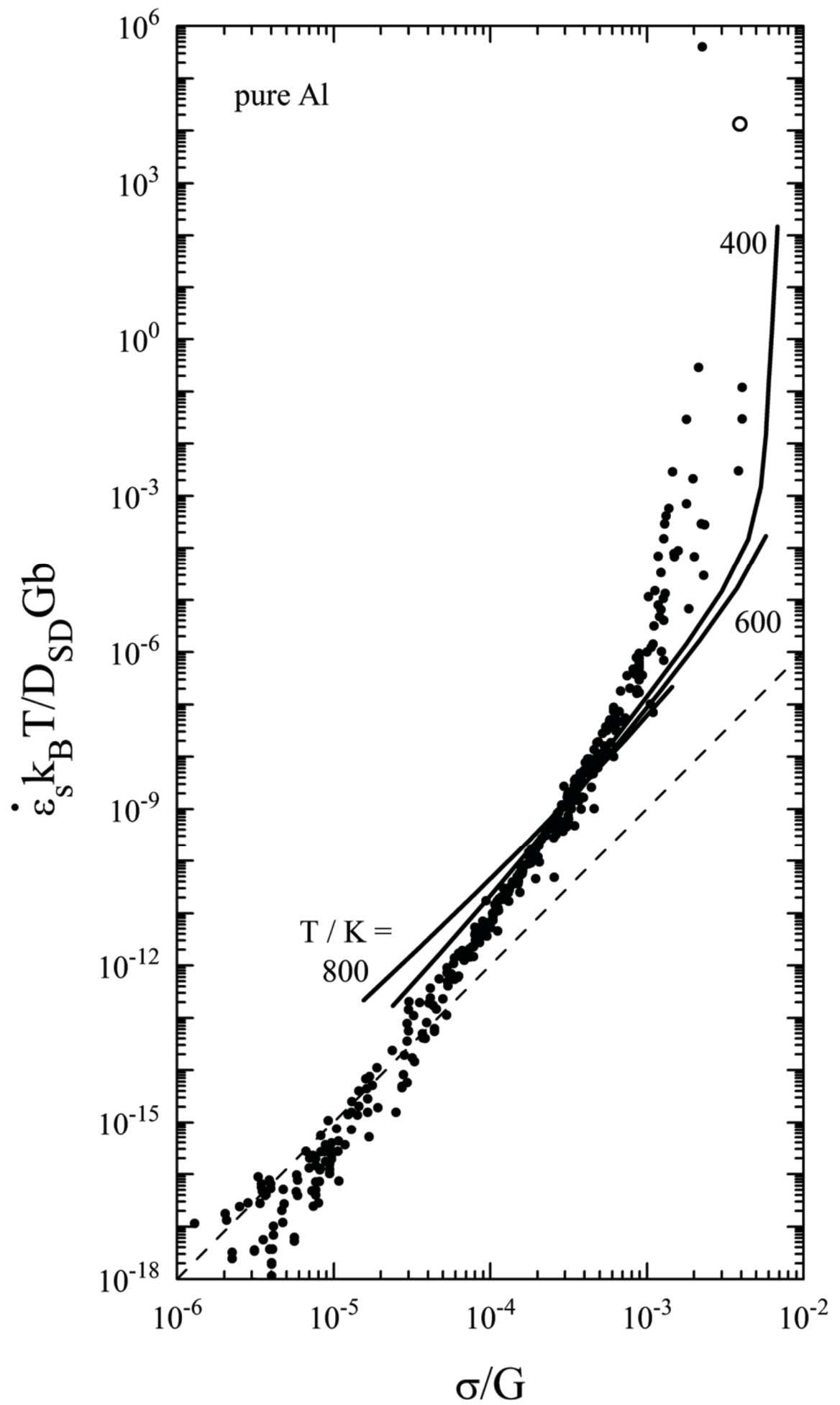

Fig. 1. Normalized steady state deformation resistance of Al. Lines from model based on $\rho_{\mathrm{r}}$-dipole annihilation. From [14]. 
Experimental data show that this point is reached rather early in a creep test, during primary creep [2]. And it is known that the creep rate of pure materials is much higher there than in the steady state $[16,2]$. Thus the simple model of steady state deformation, based on the steady state of free dislocations, is in fact in reasonable agreement with the experimental data for $\mathrm{Al}$ at low stresses in Fig. 1. The remaining deviation between observed and modeled creep rates can be explained by the fact that the currents of dislocations of opposite signs are not balanced on a local scale so that the probability of meeting a dislocation of opposite sign is less than the value of $50 \%$ assumed in the model [17], with the consequence of lower than modeled rates of formation of stable dipoles of free dislocations and of deformation at the steady state of $\rho_{\mathrm{f}}$.

As the subgrain structure forms in pure and weakly solution hardened (class M) materials, the strain rate $\dot{\varepsilon}$ decreases in creep at constant $\sigma$. This has already been reported in Garofalo's book [16] and was confirmed several times, see e.g. $[18,2,19]$. Thus the formation of subgrains leads to a shift in control of recovery rate from control by free dislocations to control by the boundaries. In fact, the low-angle boundaries constitute finely knitted nets of dislocations of the same sign, where dislocations of opposite sign are invariably caught and quickly absorbed by recombination with network dislocations or by annihilation (Fig. 2). Therefore the largest problem of free dislocations is not to dissolve at a boundary containing dislocations of opposite sign, but to reach the boundary. The latter requires that free dislocations cut those boundaries where no annihilation partner is available and continue motion to a boundary which acts as efficient sink due to its content of dislocations of opposite sign.

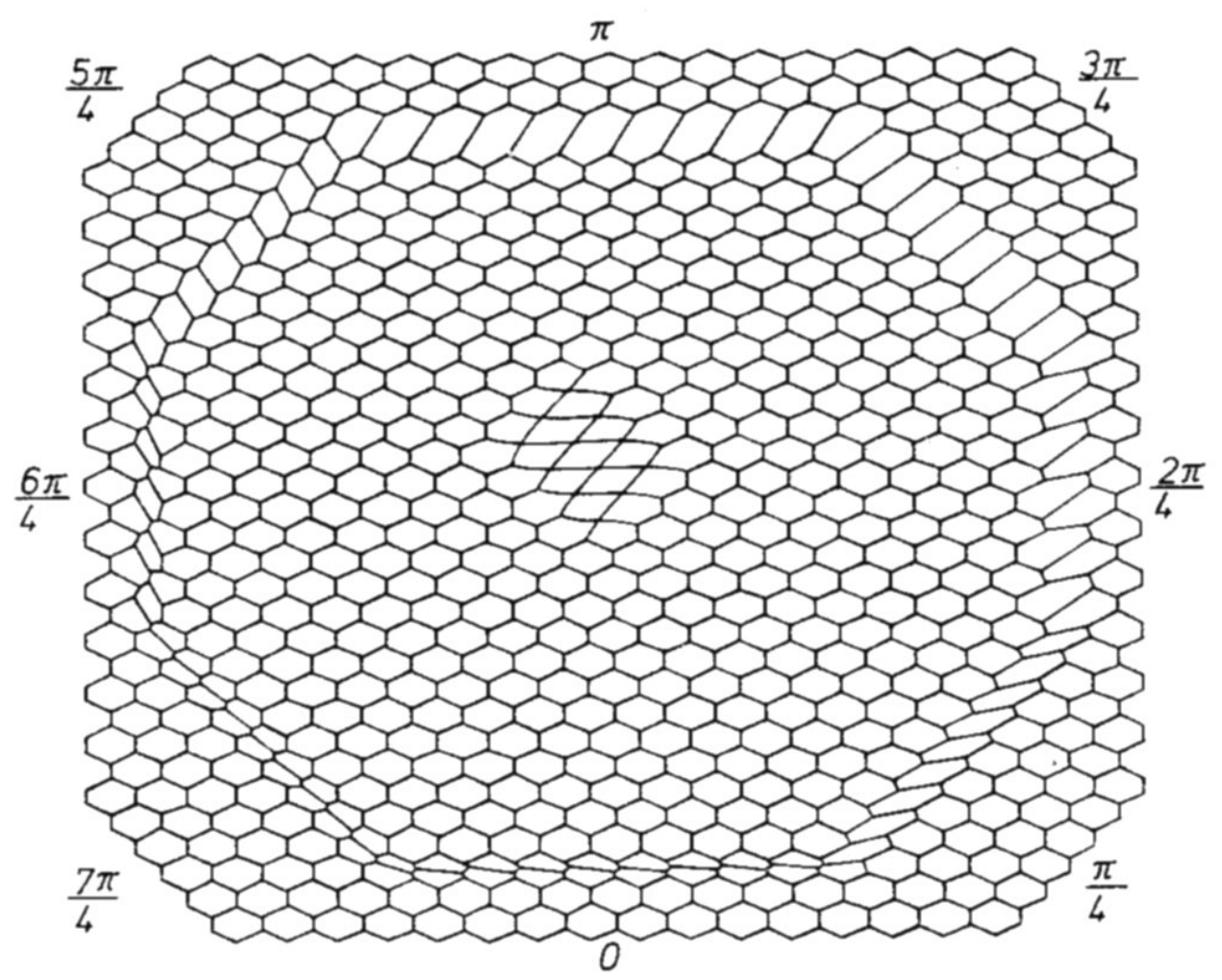

Fig. 2. A planar hexagonal dislocation network, formed from the two families of parallel dislocations shown in the center, has reacted with an intruding dislocation which has the same Burgers vector as the horizontal dislocation family, but is increasingly inclined to the horizontal by different angles in steps of $\pi / 4$ from 0 (same sign) via $\pi$ (opposite sign) to $7 \pi / 4$. Along the reaction trace the dislocation density has increased (knitting-in) or decreased (knitting-out) by different amounts depending on the inclination angle. Due to the close proximity and the multitude of reaction partners knitting is fast. From [26].

Intersection of boundaries is not easy and probably needs a local stress concentration. Stress is concentrated at the low-angle boundaries not only due to the interaction between the relatively soft 
subgrain interior with the relatively hard boundaries (composite approach [20, 21, 22]), but also because of fluctuating local stress concentrations at the boundaries caused by the migration of the boundaries [23]. If this is true, migration of the boundaries triggers continuous glide of free dislocations, making the largest contribution to creep strain. The controlling action of the boundaries is confirmed by the observation that the strain contribution caused by migrating boundaries is always a certain small fraction $(\approx 0.1)$ of the total strain [24]. Thus boundary migration not only explains the passage of free dislocations through the material, but also its recovery. When the glide of free dislocations is suppressed by stress reductions of sufficient magnitude, boundary migration continues as an autonomous process and becomes responsible for nearly all of the strain [25]. Note that this strain is distinct from the normal strain, which is associated with generation of dislocations, but is associated with recovery; thus recovery also contributes to strain $[26,11]$.

The boundary migration, however, is not only responsible for moving and annihilating free dislocations. It is also necessary to establish the steady state of the boundary structure itself [3, 24]. Boundaries of opposite dislocation content move in opposite directions and, due to their twodimensional nature, have no chance but to meet each other [27]. Therefore the migration of boundaries controls not only the kinetics of motion and recovery of free dislocations, but also the recovery of the boundary dislocations.

It follows that the rate of deformation in the steady state of creep is controlled by the boundary migration regardless of its low (direct) contribution to strain. Free dislocations need not wait for climb to reach their annihilation partner in a dipolar arrangement of parallel free dislocations. They only need to glide to an effective sink in the form of a boundary with suitable dislocation content. In this manner the boundaries not only decelerate creep as explained above for low stresses, but, by their very existence, may also accelerate the dissolution of free dislocations. The fact that the simple model of steady state creep [14] leads to steady state creep rates which at stresses above $4 \cdot 10^{-4} G$ are lower than the observed ones, is not relevant because annihilation of individual dipoles of free dislocations is not necessary, if all the dislocations have the chance of fast knitting reaction with densely knitted boundaries.

The conclusion is, that the rate of steady state deformation is completely controlled by boundary migration in the normal case of class $M$ materials. So far there is no detailed, reliable model for migration of low-angle boundaries (see, however, the discussion of a simple model in [5]). Measurements of migration rates of individual boundaries as function of stress and temperature have shown that the migration rate depends strongly on the defect content of the low-angle boundaries [28]; pure tilt boundaries migrate much faster than boundaries which have acquired some disorder by deformation [28]. A realistic model of migration of low-angle boundaries would be of great value in linking plasticity of crystals to their microstructure.

If the idea of control of steady state deformation by boundaries is correct, it must hold not only under creep conditions which have preferredly been discussed so far, but also under conditions of rate-controlled deformation. In fact, the stage IV of work hardening can be interpreted in analogy to the primary stage of creep at high homologous temperatures as the stage where boundaries take control. Also, influencing the migration rate by microstructural means, for instance pinning by precipitates, should have a strong effect on creep. This is indeed the case. Tempered martensite steels with boundaries decorated by carbonitrides are an example of particularly creep resistant materials. Elimination of the particles leads to significant increase of creep rate [29].

\section{High-angle boundaries}

What happens, if low-angle boundaries are replaced by high-angle ones? The works on ultrafinegrained materials produced by severe plastic deformation provide an answer. With increasing number of passes of equal channel angular pressing (ECAP) both the mean spacing $w$ of subgrain boundaries (low- as well as high-angle) as well as the mean spacing $d$ of high-angle boundaries saturate (see e.g. [30]). This confirms that both low-angle as well as high-angle boundaries reach a steady state under given deformation conditions; $w$ attains its steady state value distinctly earlier 
than $d$. This means that the fraction of high-angle boundaries in the subgrain structure of given size, which can well be estimated as $f_{\text {hab }}=w / d[30]$, may be varied by varying the number of passes. At the low homologous temperature of ECAP $f_{\text {hab }}$ has little influence on the deformation resistance (maximum flow stress). This can be explained by the fact that under these conditions the recovery of dislocations is athermal in nature and occurs as a byproduct of glide without the aid of thermally activated processes. At elevated temperatures and low strain rates this is no longer so. And an influence of $f_{\text {hab }}$ is clearly seen [31], most clearly in stress-controlled tests (creep) because $\dot{\varepsilon}$ is much more sensitive to microstructural changes than $\sigma$ (due to the relation $\dot{\varepsilon} \propto \sigma^{n}$ with $n>>1$. Fig. 3 is an example. The minimum $\dot{\varepsilon}_{\text {min }}$ of creep rate increases by orders of magnitude as the number of passes goes up from 2 to 8 . After the minimum the new steady state of the material (with new values of $w$ and $d$ according to the new recovery conditions) is approached. This is accompanied by work softening.

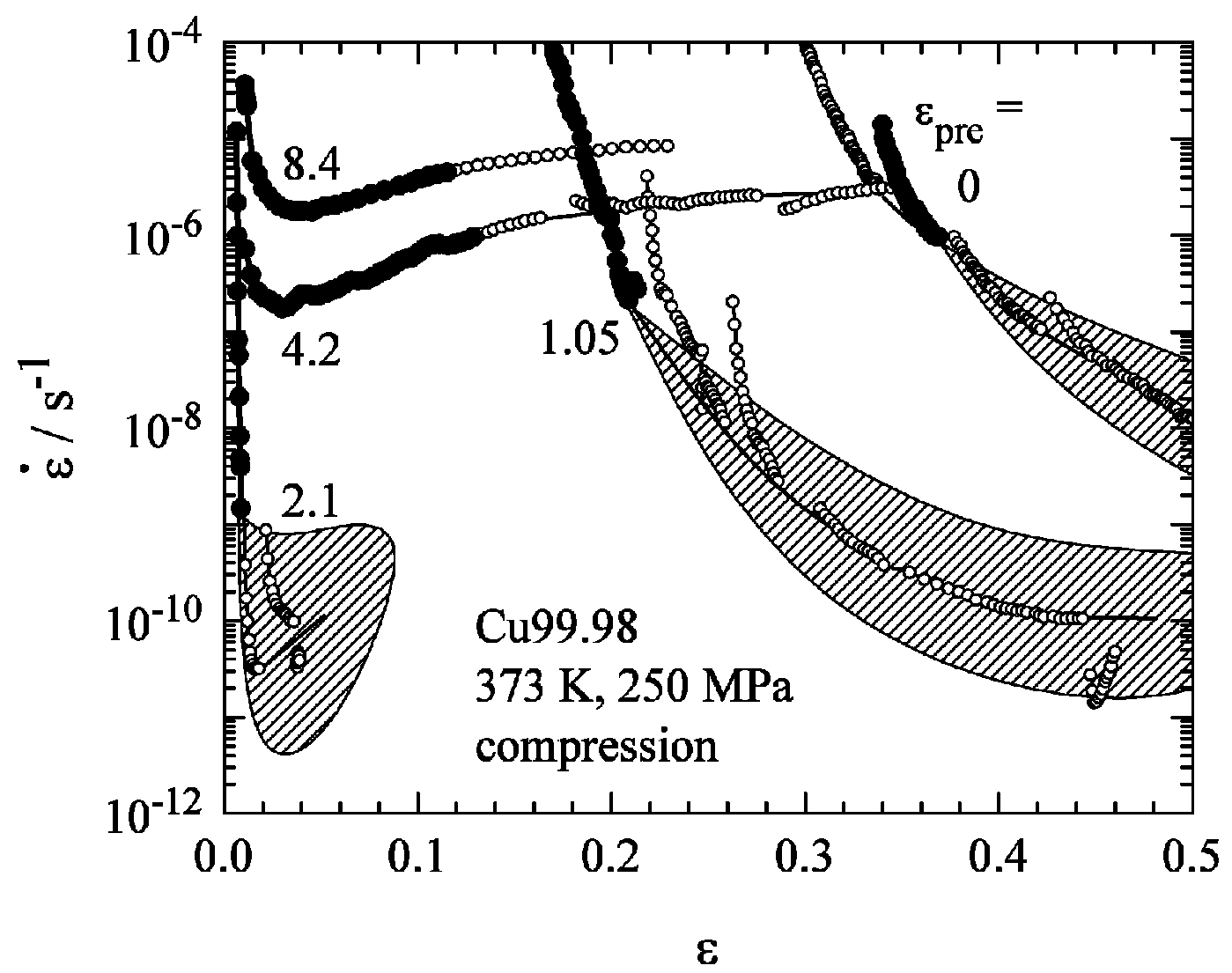

Fig. 3.Creep rate $\dot{\varepsilon}-$ strain $\varepsilon$ - curves of pure $\mathrm{Cu}$ at $373 \mathrm{~K}$ after 2 , 4 , and 8 passes of ECAP route $\mathrm{B}_{\mathrm{C}}$ corresponding to the prestrains $\varepsilon_{\text {pre. }}$ Open symbols: from stress change tests, where $\sigma=f \cdot 250 \mathrm{MPa}$ with 0.94 $\leq f \leq 1.28$, converted to $\sigma=250 \mathrm{MPa}$ using appropriate values $n=40,23,15$ of the stress exponent of creep rate in sequence of increasing $\mathcal{E}_{\text {pre }}[31]$. Dashed areas indicate maximum uncertainty.

The observed increase of $\dot{\varepsilon}_{\text {min }}$ occurs at approximately constant subgrain size $w$ so that the increase of $f_{\text {hab }}$ with decreasing $d$ is the major micro-structural change. It is a clear argument that high-angle boundaries are better sinks of dislocations than low-angle ones. This can simply be explained by diffusion being much faster along high-angle boundaries compared to diffusion at low-angle ones where lattice diffusion is controlling. However, grain boundaries do not only have a softening effect in the steady state of deformation, but a hardening as well. This leads to a distinct grain size dependence of the steady state deformation resistance in the field where $d$ is small enough to limit the mean free path of dislocations so that all the dislocations are stored at the highangle boundaries. As the mean free path scales with the steady state subgrain size $w_{\infty}=k_{\mathrm{w}} b G / \sigma$ established in large grains (where $k_{\mathrm{w}}$ is a material dependent constant in the order of 10 to 30 ), 
"small" grains in the sense defined above are characterized by $d<k^{\prime} w_{\infty}$. Considering the wide lognormal frequency distribution of grain sizes, $k^{\prime}=1$ is a reasonable value. Our consideration shows that the definition of "small" grains depends on flow stress and therefore on temperature and strain rate; higher $T$ and lower a favor "small grain" behavior.

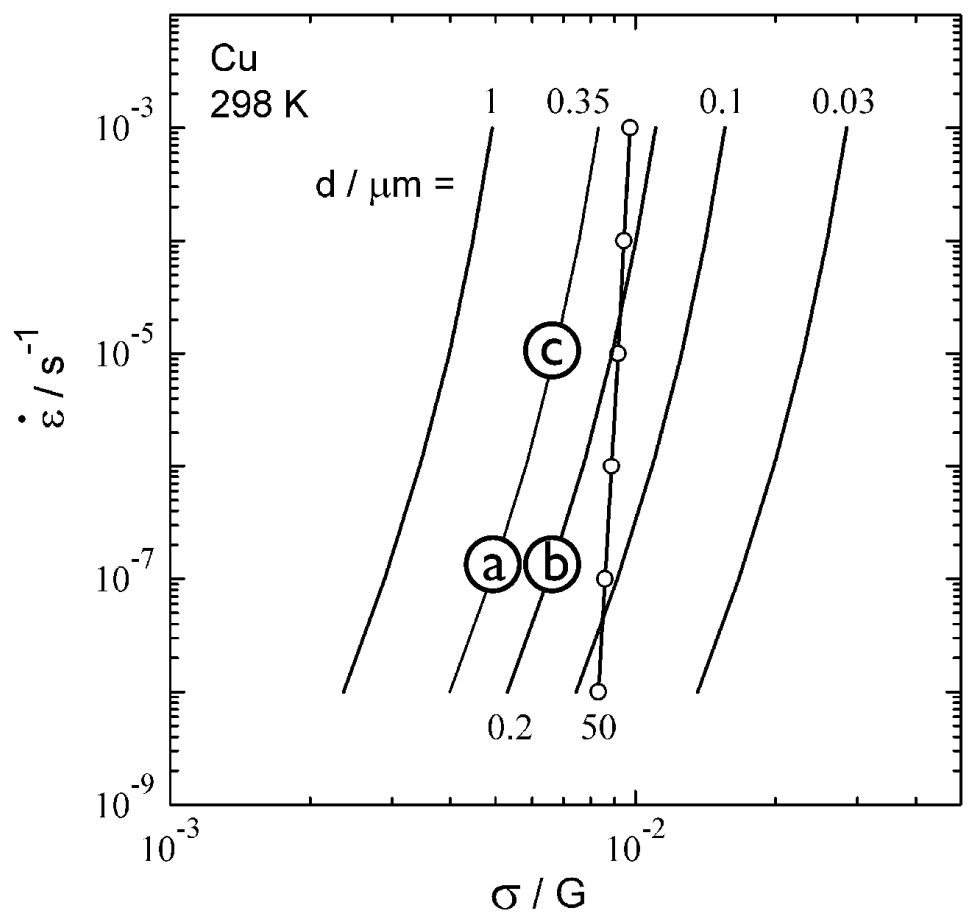

Fig. 4.Modeled steady state deformation resistance of $\mathrm{Cu}$ in dependence of grain size $d ; d=50 \mu \mathrm{m}$ : conventional grain size; $d \leq 1 \mu \mathrm{m}$ : "small" grains. From [32].

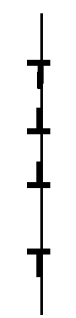

a
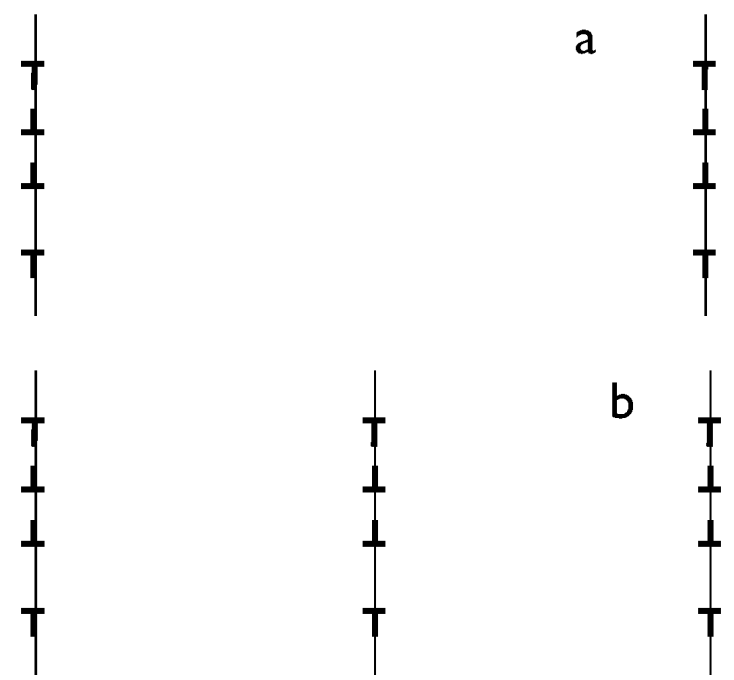

b

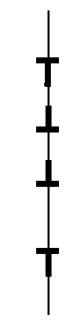

$\pm$

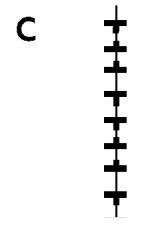

Fig. 5. Scheme of dislocation structure for cases a, b, c of Fig. 4; same $\rho$ in b and c. 
Fig. 4 illustrates the grain size dependence of the steady state deformation resistance of $\mathrm{Cu}$ with "small" grains in comparison to conventional grains. "Small" means that the dislocations are stored at the grain boundaries only, not within the grains. (To a first approximation this condition is fulfilled when the spacing of high-angle boundaries is smaller than the size $w_{\infty}$ of the subgrains in large grains.) It is seen that the steady state deformation resistance of $\mathrm{Cu}$ with "small" grains may be lower or higher than that of $\mathrm{Cu}$ with conventional grains depending on grain size and deformation conditions. The curves for small grains were calculated by Zeng [32] under the following assumptions: i) the average density $\rho$ of dislocations (mainly stored at high-angle boundaries) determines the deformation resistance $\sigma=\alpha M G b \rho^{0.5}$ where $\alpha=0.2$ is the dislocation interaction constant and $M=3$ is the Taylor factor. ii) All dislocations are in dipolar configuration and dissolve by climb or by spontaneous annihilation. iii) $d$ remains constant during deformation. iv) Strain contributions from grain boundary sliding and Coble creep are negligible.

The result for small grains can be explained as follows. The rate of local recovery at individual grain boundaries depends solely on the local areal dislocation density at grain boundaries (length per boundary area). Constant $\dot{\varepsilon}$ means constant areal storage rate of dislocations at high-angle boundaries independent of grain size. In the steady state this areal storage rate is compensated by an areal recovery rate of equal magnitude. This is achieved when the areal density of dislocations at boundaries is he same independent of $d$. Decreasing $d$ therefore means increasing volumetric density $\rho$, hence higher flow stress (transition from a to $\mathrm{b}$ in Figs. 4 and 5). Increasing $d$ at constant $\sigma$, i.e. constant $\rho$, means increase of areal density of boundary dislocations, hence higher rate of recovery and higher $\dot{\varepsilon}$ (transition from $\mathrm{b}$ to $\mathrm{c}$ in Figs. 4 and 5). The model predicts $\sigma \propto d^{-0.5}$ at constant $\dot{\varepsilon}$ (Hall Petch type relationship) and $\dot{\varepsilon} \propto \sigma^{8}$ for climb-controlled recovery [32]. The curvature of the lines in Fig. 4 results from the increase of the contribution of athermal recovery.

If the temperature gets high enough for sliding and migration of high-angle boundaries to occur at high rates, new mechanisms of recovery of dislocations at high-angle boundaries are opened in addition to dipole annihilation. This leads to additional softening with the potential consequence of superplasticity, unless the grain structure is replaced by recrystallization [33].

In conclusion we state that high-angle boundaries do influence the deformation resistance by enhancing generation of dislocations as well as thermally activated recovery, provided that the dislocations are stored at the high-angle boundaries only. As soon as a subgrain structure establishes within the grains because the mean free path of dislocations is distinctly smaller than the grain size, the low-angle boundaries take control and the dependence on grain size disappears. As the steady state subgrain size is a unique function of the applied stress, there is no explicit dependence of the steady-state creep rate on subgrain size in coarse-grained materials. However, the variation of subgrain size in the transient in between different steady state leads to variation of the deformation resistance during the transients.

\section{Phase boundaries}

Phase boundaries differ from grain boundaries in that they generally separate materials with significantly different deformation resistance. This has a simple, but important consequence. Dislocations of a given sign arrive at the boundary when the soft phase deforms. But these dislocations have no chance to find an annihilation partner if the neighboring phase is hard and does not deform. Recovery at the phase boundary of the type occurring at the grain boundaries as considered above is impossible, because there are no dipoles available (i.e., no slipped areas which may coalesce). The only ways for the dislocations to recover are to move around the hard phase or wait until the stress in the hard phase is high enough for deformation to set in.

The mechanism of suppression of recovery and increasing the steady state deformation resistance is acting for instance in Ni-base superalloys and in ferritic steels. In the superalloys dislocation networks in the phase boundaries play a significant role. These networks, however, are not of the type of low-angle boundaries, but have the character of geometrical dislocations (misfit 
dislocations) which relieve the coherency stresses between the coherent $\gamma$ - and $\gamma^{\prime}$-phases. The networks form additional obstacles to penetrating the hard $\gamma^{\prime}$-particles. This explains why the creep resistance of superalloys increases when the lattice misfit (at test temperature!) increases [34]. The steady state creep rate is controlled at high temperatures by the bypassing of the hard phase and at low temperatures, where this process is too slow, by the onset of deformation in the hard phase under the influence of concentrated stress. For the sake of simplicity the difference between the phase boundaries parallel and perpendicular to the stress axis were neglected (without imparting the validity of the main arguments).

In drawn wires of ferrite-cementite structures the cementite particles form a lamellar structure. The lamellae are so long that they cannot be circumvented by the dislocations at low temperatures. As the cementite particles are so hard that they do not deform, the same mechanism of suppression of recovery due to lack of dipoles is present as described in the preceding example; the dislocations of a given Burgers vector $\mathbf{b}$ cannot recover as they all have the same sign. The result is a tremendous increase of dislocation density and strength during straining, for instance by wire drawing. Strengths of $5 \mathrm{GPa}$ have been reported [35]. The defect energy stored at the phase boundaries is so large that relaxation of the stresses by amorphization of the phases sets in.

\section{Summary}

Our considerations have shown that crystal boundaries play an essential role in determining the strength and the deformability of crystalline materials. Usually in single phase materials the boundaries are of low-angle type. They control the steady state of deformation, i.e., the maximum of deformation resistance at a given temperature and type of deformation, by their migration which not only triggers the motion of the free dislocations making the largest strain contribution, but also is necessary to maintain the steady state of the boundary structure. High-angle boundaries take over in control of steady state deformation resistance, if their spacings become so small that dislocations are no longer stored within the grains. The high-angle boundaries strengthen or soften the material in comparison to material with conventional grain size depending on whether the enhancement of storage of the dislocations at the boundaries or enhanced recovery by fast diffusion at the boundaries is the dominant effect. Phase boundaries of hard particles may suppress or block recovery by hindering formation of dislocation dipoles, which may lead to significant increase of strength.

\section{Acknowledgements}

Thanks are due to the Deutsche Forschungsgemeinschaft for funding the project BL135/29 and the Office of Naval Research Global - Department of the Navy, Science \& Technology, for partially supporting participation in this conference under Grant N00014-08-1-1011.

\section{References}

[1] J. Kratochv1l and M. Saxlova: Scripta Metall. Mater. Vol. 26 (1992), p. 113.

[2] W. Blum in: "High-Temperature Deformation and Creep of Crystalline Solids". In Plastic Deformation and Fracture of Materials, H. Mughrabi, editor, vol. 6 of Materials Science and Technology, ed. R. W. Cahn, P. Haasen and E. J. Kramer, Weinheim: VCH Verlagsgesellschaft, (1993), p. 359-405.

[3] E. Nes: Progr. Mater. Sci. Vol. 41 (1998), p. 129.

[4] W. Blum, P. Eisenlohr and F. Breutinger: Metall. Trans. Vol. 33A (2002), p. 291. 
[5] W. Blum, S. Straub and S. Vogler: Creep of pure materials and alloys“, Proc. 9th Int. Conf. on the Strength of Metals and Alloys (ICSMA 9), ed. D. G. Brandon, R. Chaim, and A. Rosen, Vol. I, London: Freund Publishing House( 1991), p. 111-126.

[6] W. Blum in: "Creep Simulation", Continuum Scale Simulation of Engineering, Materials Fundamentals - Microstructures - Process Applications, ed. L.-Q. Chen, F. Barlat, F. Roters, and D. Raabe, Weinheim: Wiley-VCH (2004), p. 607-620.

[7] R. Sedlacek, W. Blum, J. Kratochvil and S. Forest: Metall. Trans. A, Vol. 33 (2002) p. 319.

[8] W. Blum and H. J. McQueen in: "Dynamics of recovery and recrystallization", Aluminium Alloys, Their Physical and Mechanical Properties - Proceedings ICAA5, ed. J. H. Driver, B. Dubost, F. Durand, R. Fougeres, P. Guyot, P. Saintfort, and M. Suery, Part 1, vol. 217-222 of Materials Science Forum, Zuerich, Switzerland: Transtec Publications (1996), p. 31-42.

[9] M. Winning, G. Gottstein and L.S. Shvindlerman: Mater. Sci. Eng., Vol. A317 (2001), p. 17.

[10]T. G. Langdon: Z. Metallkd. Vol. 96 (2005), p. 522.

[11]U.F. Kocks and H. Mecking: Progr. Mater. Sci. Vol. 48 (2003), p. 171.

[12]B. Holmedal, K. Marthinsen and E. Nes: Z. Metallkd. Vol. 96 (2005), p. 532.

[13]P. Eisenlohr in: "On the role of dislocation dipoles in unidirectional deformation of crystals", Dr.-Ing. thesis, University of Erlangen-Nürnberg, (2004).

[14]P. Eisenlohr and W. Blum: Mater. Sci. Eng. A, Vol. 400-401 (2005), p. 175.

[15]H. J. Frost and M. F. Ashby in: Deformation-Mechanism Maps. Oxford: Pergamon Press, (1982).

[16]B. Garofalo in: Fundamentals of Creep and Creep Rupture. McMillan, New York, (1965).

[17]P. Eisenlohr and W. Blum: accepted by Mater. Sci. Eng. A, (2008).

[18] W. Blum, A. Absenger and R. Feilhauer in: "Dislocation Structure in polycrystalline AlZn during transient and steady state creep, Proc. 5th Int. Conf. on the Strength of Metals and Alloys (ICSMA 5), ed P. Haasen, V. Gerold, and G. Kostorz, Oxford: Pergamon Press (1980), p. 265-270.

[19]P. Sadrabadi, P. Eisenlohr, G. Wehrhan, J. Stäblein, L. Parthier and W. Blum: accepted by Mater. Sci. Eng. A, (2008).

[20]H. Mughrabi, Acta metall., Vol. 31 (1983) p. 1367.

[21]S. Vogler and W. Blum in: "Micromechanical modelling of creep in terms of the composite model“", Creep and Fracture of Engineering Materials and Structures, ed B. Wilshire and R.W. Evans, London: The Institute of Metals (1990), p. 65.

[22]R. Sedlacek and W. Blum: Comp. Mater. Sci. Vol. 25 (2002), p. 200.

[23]D. Caillard: Philos. Mag. A, Vol. 51 (1985), p. 157.

[24]M. Biberger and W. Blum: Phil. Mag. A Vol. 66 (1992), p. 27.

[25]W. Müller, M. Biberger and W. Blum: Phil. Mag. A Vol. 66 (1992), p. 717.

[26]W. Blum: Scripta Metall. Vol. 18 (1984), p. 1383.

[27]M. Biberger and W. Blum: Phil. Mag. A Vol. 65 (1992), p. 757.

[28]M. Winning: Scripta Mater. Vol. 58 (2008), p. 85.

[29]A. Kostka, K.-G. Tak, R.J. Hellmig, Y. Estrin and G. Eggeler: Acta Mater. Vol. 55 (2007), p. 539. 
[30] M. Cabibbo, W. Blum, E. Evangelista, M.E. Kassner and M.A. Meyers: Metall. Mater. Trans. A Vol. 39 (2008), p. 181.

[31]W. Blum, P. Eisenlohr, and V. Sklenicka in: "Creep of bulk nanostructured materials“, Bulk Nanostructured Materials, ed. M. Zehetbauer and Y. T. Zhu, Weinheim: Wiley-VCH, in print.

[32]X.H. Zen in: "Statistical dislocation models of plastic deformation of crystalline materials", Dr.-Ing. thesis, University of Erlangen-Nürnberg, (2007).

[33]Y.J. Li, R. Kapoor, J.T. Wang and W. Blum: Scripta Mater. Vol. 58 (2008) p. 53.

[34]F. Pyczak, S. Neumeier and M. Göken: accepted by Mater. Sci. Eng. A (2008).

[35]S. Goto in: “Application of cold drawn pearlitic microstructure for development of ultra-high strength steel wires“. Seminar at University of Erlangen-Nürnberg (2007). 